\title{
Informationsdienst Wissenschaft
}

\author{
Press release
}

\author{
Neues Fischereimanagement soll Europas Fischbestände \\ sichern \\ Dr. Andreas Villwock Pressestelle \\ Leibniz-Institut für Meereswissenschaften, Kiel
}

$10 / 15 / 201012: 09$

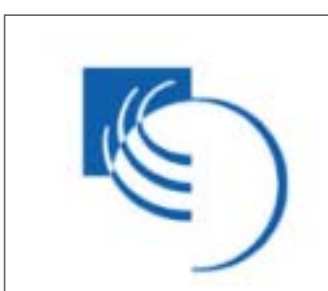

IFM-GEOMAR

Immer wieder warnen die Kieler Meereswissenschaften vor den Konsequenzen einer Überfischung der Meere. Bereits heute gelten drei Viertel der marinen Fischbestände weltweit als überfischt, in Europa sind es laut EU Kommission sogar 88 Prozent. Kieler Wissenschaftler haben jetzt Konzepte entwickelt, die den Zusammenbruch der Bestände verhindern können und der Fischerei gleichzeitig bessere Verdienstmöglichkeiten bieten. Die Studie erscheint in der aktuellen Ausgabe der international führenden Fachzeitschrift Fish and Fisheries.

Lange galt Fisch als verlässliche Nahrungsquelle. Die Ozeane sind groß, und die Bestände der in ihnen lebenden Fische schienen unerschöpflich. Doch seit Jahrzehnten wird mehr Fisch aus dem Meer geholt als nachwachsen kann. Das Ende ist absehbar: Fischarten wie Scholle, Hering oder Kabeljau sind in einigen Regionen schon jetzt fast verschwunden. Wissenschaftler des Kieler Leibniz-Instituts für Meereswissenschaften (IFM-GEOMAR) und des Exzellenzclusters

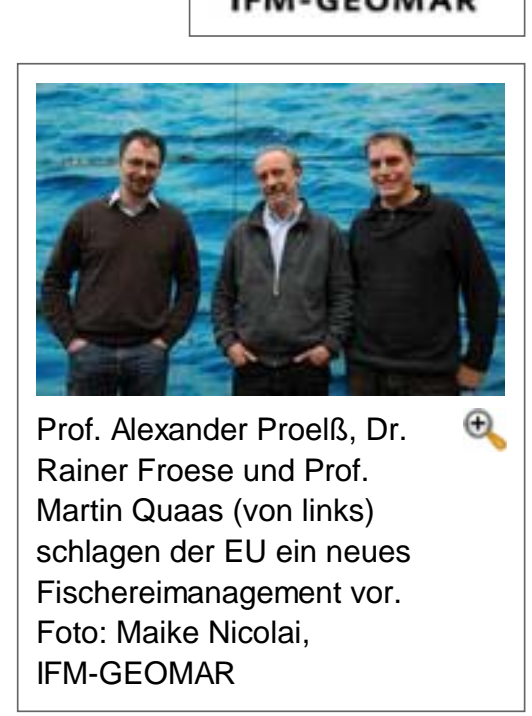
„Ozean der Zukunft" haben ein neues Konzept erarbeitet, das Strategien einer nachhaltigen Sicherung der Fischbestände Europas und eine Erholung bereits stark dezimierter Arten aufzeigt. Der Artikel „Generic harvest control rules for European fisheries“ erscheint in dieser Woche in der führenden Fachzeitschrift Fish and Fisheries. Mit dem darin vorgeschlagenen Managementplan kann es gelingen, gefährdete Bestände zu stabilisieren und wieder aufzustocken. Dies würde langfristig 60 Prozent höhere Fänge von viermal größeren Beständen erbringen. Der Kieler Vorschlag unterscheidet sich stark von dem gegenwärtigen Plan der Kommission, der Fischerei auch bei sehr kleinen Bestandsgrößen und Fänge weit über dem maximalen nachhaltigen Ertrag erlaubt. Der Managementplan der Kieler Wissenschaftler sieht auch erstmals einen Sicherheitsspielraum für die Bestände und eine Gewinnmaximierung für die Fischer vor.

Der Vorschlag für ein neues Fischereimanagement in Europa entstand in enger interdisziplinärer Zusammenarbeit von Experten der Wirtschaftswissenschaften, des Internationales Seerecht sowie der Marinen Ökologie aus Kiel, Rostock, Washington und Tasmanien. Dr. Rainer Froese, Fischereibiologe am IFM-GEOMAR, sieht einen Grund für das zukunftsweisende Ergebnis in der interdisziplinären Zusammenarbeit: „Wir haben in den Vorschlag die besten internationalen Erfahrungen mit Fischereimanagement eingebaut." Auch Prof. Martin Quaas, Direktor des Instituts für Volkswirtschaftslehre der Christian-Albrechts Universität zu Kiel (CAU) und Leiter der Umwelt-, Ressourcen- und Ökologischen Ökonomik bestätigt: „In unserem Vorschlag haben wir ökologische und ökonomische Ziele zusammengebracht." Prof. Alexander Proelß, Leiter der Abteilung Seerecht am Walther-Schücking-Institut für Internationales Recht an der CAU betont: „Mit unserem Vorschlag würde endlich das bereits existierende internationale Seerechtsübereinkommen aus dem Jahre 1982 umgesetzt.“

Der Vorstoß des IFM-GEOMAR sowie des Exzellenzclusters „Ozean der Zukunft“ findet auch politischen Rückhalt. „Die Studie liefert einen interessanten Diskussionsbeitrag, und sie ist es wert, dass sich auch der Internationale Rat für Meeresforschung damit befasst.", urteilt Bundestagsmitglied Holger Ortel (SPD). Die Umweltverbände zeigen sich ebenfalls positiv beeindruckt. So sagt Dr. Iris Menn, Meeresbiologin bei Greenpeace: „Viermal größere Bestände sind ein wesentlicher Schritt zum Wiederaufbau unserer marinen Ökosysteme."

Wenn sich alle einig sind, fehlt nur noch die Umsetzung des Konzeptes. Ob dies im europäischen Kontext auch zu verwirklichen ist, wird die Zukunft zeigen. 


\section{Originalarbeit:}

Rainer Froese, Trevor A. Branch, Alexander Proelß, Martin Quaas, Keith Sainsbury, Christopher Zimmermann: Generic Harvest Control Rules for European Fisheries. Fish and Fisheries. DOI:

10.1111/j.1467-2979.2010.00387.x

Bildmaterial:

Unter www.ifm-geomar.de/presse steht Bildmaterial zum Download bereit.

Ansprechpartner:

Dr. Rainer Froese, IFM-GEOMAR, Marine Ökologie, Tel. 0431 600-4579, rfroese@ifm-geomar.de Prof. Alexander Proelß, Leiter der Abteilung Seerecht am Walther-Schücking-Institut für Internationales Recht an der CAU, Tel. 0431 880-2083, aproelss@internat-recht.uni-kiel.de

Prof. Martin Quaas, Direktor des Instituts für Volkswirtschaftslehre der CAU und Leiter der Umwelt-, Ressourcen- und Ökologischen Ökonomik, Tel. 0431 880-3616, quaas@economics.uni-kiel.de Maike Nicolai (Öffentlichkeitsarbeit IFM-GEOMAR), Tel. 0431 600-2807, mnicolai@ifm-geomar.de

URL of this press release: http://idw-online.de/pages/en/news391935

\section{Criteria of this press release:}

Animal sciences / Agriculture / Forestry, Environment / Ecology, Nutrition / Health / Health Care, Sea / Climate transregional, national

Research results, Transfer of Science or Research German

(C) 1995-2010 Informationsdienst Wissenschaft e. V. 